\title{
PROCESSO DE ENFERMAGEM: VIVÊNCIA NA IMPLANTAÇÃO DA FASE DE DIAGNÓSTICO*
}

[The nursing process: experience in the implementation of the diagnostic phase]

[ $P$ roceso de enfermería: experiencia en la implantación de la fase de diagnóstico]

\author{
D aniele L aís B randalize** \\ Carmen Elisabeth K alinowski***
}

\begin{abstract}
RESUM O: Trata-se de um estudo qual itativo, que retrata uma observação sistemática, participante, individual e em campo, relativo à aplicação do processo de enfermagem pelos enfermeiros e equipe de enfermagem, com proposta de implantação da fase de diagnóstico. Ocorreu durante a disciplina de Estágio Supervisionado II de um curso de graduação em enfermagem, em duas U nidades de Terapia Intensiva de um Hospital U niversitário na cidade de Curitiba, no período de fevereiro a junho de 2004. A pesquisa mostrou que o processo é aplicado, mas de forma incompleta e desarticulada. Verificou-se também que a prescrição não é vista pela equipe de enfermagem como orientadora e que não há dependência dessa para a real ização da assistência. Percebeu-se a presença de barreiras que impedem a implementação do processo e que precisam ser transpostas, como a inadequação quanto ao quantitativo de enfermei ros estabelecido pelo Conselho Federal de Enfermagem. A pós anál ise dos dados, decidiu-se pela implantação da fase de diagnóstico e reformulação do impresso de histórico. Realizou-se também um treinamento com os enfermeiros e um manual da sistematização da assistência foi construído. Percebeu-se dificuldades dos enfermeiros na aplicação do impresso de diagnóstico e espera-se que, para tentar superálas, estes continuem com as discussões, visando não somente preencher o papel, mas saber articulá-lo no processo. A credita-se que este seja mais um passo da caminhada rumo à melhor qualidade da assistência prestada ao ser humano e conseqüente crescimento profissional para a enfermagem, com val orização e autonomia.
\end{abstract}

PALAVRAS-CHAVE: Processo de Enfermagem; Implantação; Diagnóstico de Enfermagem.

\footnotetext{
* M onografia de Conclusão de Curso de Graduação em Enfermagem da Universidade Federal do Paraná. 2004.

**Enfermeira, especializanda em Enfermagem em Terapia Intensiva, PUC-PR; mestranda em Enfermagem, UFPR.

***Professora do Departamento de Enfermagem da UFPR.

Orientadora.
}

\section{INTRODUÇÃO}

Desde Florence Nightingale há uma preocupação com a identidade profissional da enfermagem. Inicialmente os cuidados começaram a ser organizados de forma funcional, importando-se o cumprimento de tarefas, a realização de procedimentos técnicos e atividades rotineiras. Depois surgiram os estudos de caso e os planos de cuidados, utilizados pela primeira vez no Brasil em 1934, como uma forma de organização e individualização do cuidado de enfermagem ${ }^{(1)}$.

A partir da década de 60 do século passado, houve uma mai or ênfase na observação dos aspectos interpessoais, intel ectuais e científicos da enfermagem e a expressão processo de enfermagem passou a definir a situação desta profissão em três aspectos básicos: o comportamento do paciente, a reação do enfermeiro e as ações da enfermagem que são destinadas ao bem estar do paciente ${ }^{(1)}$.

0 processo de enfermagem passou a ser empregado como um instrumento de trabal ho do enfermeiro, como um método sistematizador da sua assistência, que possibilita ao enfermeiro aplicar na prática o marco teórico que direciona e que embasa as suas ações. A o mesmo tempo os enfermeiros buscavam a sua autonomia profissional e 0 status da profissão. Neste sentido, o processo de enfermagem foi definido como

a dinâmica das ações sistematizadas e inter-relacionadas, que visam a assistência ao ser humano e caracteriza-se pelo interrelacionamento e dinamismo de suas fases ou passos ${ }^{(2: 35)}$.

Desde que entrei em contato pela primeira vez com tema em sala de aula, o processo de enfermagem sempre me chamou a atenção. Por ser considerado como uma importante ferramenta para o trabal ho e também para a visi bilidade do enfermeiro nas instituições, procurei observar e conhecer como ele se aplicava na prática.

A o conhecer diferentes experiências sobre a aplicação do processo no cotidiano do trabal ho dos enfermeiros, observei a dicotomia entre o ensinado e o realizado. A partir destas experiências, surgiu a necessidade de realizar um 
estudo mais aprofundado sobre a aplicação do processo de enfermagem.

R efletindo sobre essa realidade vivenciada durante a graduação e tendo como exigência para a conclusão do curso de graduação em enfermagem o desenvolvimento de uma monografia, decidi aproveitar o local onde aconteceram as atividades de estágio final para realizar este estudo e contribuir no que se refere ao tema com a U nidade escoIhida, considerando-se que, no local, os profissionais já desenvolviam todas as etapas do processo, exceto a fase do diagnóstico. Dessa forma, o desafio deste trabalho foi a reelaboração da fase do histórico e a implantação da fase do diagnóstico.

\section{REVISÃO DE LITERATURA}

A s mudanças capazes de exercer influência na prestação de cuidados ao paciente têm sido relatadas em várias partes do mundo. U m importante marco foi registrado em 1992 com a publicação da normatização nacional sobre a obrigatoriedade da presença do enfermeiro em todas as unidades de serviço onde são desenvolvidas ações de enfermagem, durante todo o período de funcionamento da instituição de saúde ${ }^{(3)}$. Como arte e ciência não-absoluta, a enfermagem vem hoje procurando demonstrar a importância do cuidado na assistência de saúde e segue em busca de melhoria.

A enfermagem é uma arte que implica no cuidado dos pacientes durante 0 adoecimento e 0 ajuda para alcançar um potencial de saúde máximo ao longo de seu ciclo vital. 0 enfermeiro se esforça por adaptar-se às necessidades dos indivíduos dentro das diversas situações, mediante a relação pessoal com o indivíduo, os familiares e as comunidades. A ciência de enfermagem se baseia em um amplo marco teórico. 0 processo de enfermagem é um método mediante o qual se aplica este marco à prática de enfermagem. 0 principal propósito do processo de enfermagem é proporcionar a possibilidade da visualização ou da aplicação de um marco teórico dentro do qual se podem satisfazer as necessidades individual izadas do paciente ${ }^{(4)}$.

A primeira fase do processo, o histórico de enfermagem, é definida por Horta ${ }^{(2: 41)}$ como "o roteiro sistematizado utilizado para o levantamento de dados do ser humano (significativos para o enfermeiro) que tornam possível a identificação de seus problemas". Existem vários tipos de abordagem no exame físico, sendo que as duas mais comumente usadas são a abordagem cefal ocaudal e a abordagem dos sistemas corporais ${ }^{(5)}$. magem, é

A segunda fase do processo, o diagnóstico de enfer-

a identificação das necessidades básicas do ser humano que precisam de atendimento e a determinação, pela enfermeira, do grau de dependência deste atendimento em natureza e extensão (2:35)
A pós analisar os dados levantados no histórico de enfermagem, 0 enfermei ro realiza jul gamentos relativos a esses dados, que são os diagnósticos de enfermagem, visando alcançar ou manter o estado de saúde desejado ${ }^{(6)}$.

Em 1990, a North A merican Nursing Diagnosis A ssociation (NANDA), propôs a seguinte definição:

o diagnóstico de enfermagem é um julgamento clínico sobre as respostas, atuais ou potenciais, que indivíduos, famílias ou comunidades apresentam a problemas de saúde/processos de vida.

0 principal trabalho da NA NDA é direcionar a padronização de linguagem dos diagnósticos e na sua classificação constam todos os diagnósticos já aceitos, agrupados segundo os Padrões de Respostas Humanas ${ }^{(1: 65)}$, que são: trocar, comunicar, relacionar, val orizar, escolher, mover, perceber, conhecer e sentir ${ }^{(7)}$. A designação de Padrão de R espostas Humanas regeu a classificação da N A NDA e fundamentou-se na compreensão de que todas as categorias diagnósticas expressam respostas humanas e que conjuntos de diagnósticos que compartilham al gumas propriedades constituem padrões ${ }^{(1)}$.

A terceira fase do processo de enfermagem, o plano assistencial, é "a determinação global da assistência de enfermagem que o paciente deve receber diante do diagnóstico estabelecido" (2:36). 0 enfermeiro prioriza os problemas levantados, identifica resultados ou metas mensuráveis, sel eciona intervenções adequadas e documenta o plano de cuidados. Como nem todos os problemas dos pacientes podem ser resolvidos durante um período normalmente curto de hospital ização, éimportante determinar quais problemas requerem maior atenção. Isso pode ser feito por meio do estabelecimento de prioridades. A priorização envolve uma classificação dos problemas desde o de maior importância até 0 de menor significação ${ }^{(8)}$.

A prescrição de enfermagem, quarta fase do processo de enfermagem, foi definida como a

implementação do plano assistencial pelo roteiro diário que coordena a ação da equipe de enfermagem na execução dos cuidados adequados ao atendimento das necessidades básicas e específicas do ser humano ${ }^{(2: 36)}$.

Este roteiro é avaliado continuamente, fornecendo os dados necessários para o próximo passo.

Na quinta fase do processo, a evolução de enfermagem, que consiste no relato diário das mudanças sucessivas que ocorrem no ser humano; é uma avaliação global do plano de cuidados, pois, da evolução poderão surgir mudanças no diagnóstico, no plano assistencial e na prescrição, visando melhorar a assistência de enfermagem implementada ${ }^{(2)}$.

O prognóstico, sexta e última fase do processo,

é a estimativa da capacidade do ser humano em atender a

Cogitare Enferm 2005 set/dez; 10(3):53-7 
suas necessidades básicas após a implementação do plano assistencial e à luz dos dados fornecidos pela evolução de enfermagem. 0 prognóstico indicará as condições que o paciente atingiu na alta médica [...]. U m bom prognóstico é aquele que leva ao autocuidado, portanto, à independência de enfermagem; um prognóstico sombrio é aquele que se dirige à dependência total (2:68)

\section{METODOLOGIA}

Trata-se de um estudo com abordagem qualitativa, que descreve os passos na reconstrução da etapa do histórico com a inserção da fase do diagnóstico de enfermagem. Iniciou-se pela observação, durante o primeiro mês de estudo, das atividades diárias dos enfermeiros (2) e equipe de enfermagem (18) do turno da manhã, a qual foi sistematizada e esquematizada após discussão com os mesmos.

Desta observação foi apontada a necessidade de implantar a fase de diagnóstico. A pós reunião com os enfermeiros da unidade, optou-se em reelaborar a primeira fase do processo, o histórico, procurando-se fazer a relação entre o levantamento de dados e o diagnóstico. Também foi elaborado uma relação de diagnósticos com relação às situações de maior freqüência de internação nas unidades de terapia intensiva. A seguir foi el aborado o exame físico utilizando a abordagem cefalocaudal e dos sistemas corporais. A pós a confecção do impresso, este foi testado pelos enfermeiros assistenciais das Unidades de Terapia Intensiva para que os mesmos posteriormente dessem continuidade às discussões e reformulações que se fizessem necessárias.

0 estudo ocorreu durante a disciplina de Estágio Supervisionado II de um curso de graduação em enfermagem, em duas uni dades de terapia intensiva de um $\mathrm{H}$ ospital U niversitário na cidade de Curitiba, no período de fevereiro a junho de 2004. Para a real ização do estudo, solicitouse permissão ao enfermeiro gestor destas unidades, atendendo a Resolução 196/96 (9).

\section{ANÁLISE DOSDADOS}

Verificou-se que o processo de enfermagem era aplicado, mas de forma incompleta (sem a fase de diagnóstico de enfermagem) e desarticulada em suas fases. Percebeuse que a prescrição de enfermagem não era vista pela equipe de enfermagem como orientadora do cuidado, sendo que não havia dependência dessa para a real ização da assistência. A justificativa por eles relatada foi que os cuidados prescritos não são individualizados, isto é, na maioria das vezes as prescrições de enfermagem são generalizadas e repetitivas, comuns a todos os pacientes e que os cuidados específicos não são prescritos pelos enfermeiros.

0 modelo conceitual adotado que embasou a realização deste estudo, foram a Teoria das Necessidades $\mathrm{Hu}$ manas B ásicas eno referencial de Wanda de A guiar $\mathrm{H}$ orta ${ }^{(2)}$.

Um dos desafios deste trabalho esteve na operacional ização destes model os no contexto da prática, pois os mesmos seguem caminhos diferentes na sua construção, sendo que Wanda Horta trabalha com Necessidades H umanas B ásicas e a N A N DA com os Padrões de Respostas Humanas. 0 grande desafio foi tentar unir estes dois referenciais. Sendo assim, tentou-se trabalhar com os conceitos de Horta na estrutura do processo como um todo e adaptar o modelo da NA N DA no Histórico e Diagnóstico de Enfermagem (1).

U ma condição verificada e que poderia estar dificultando a implementação do processo de enfermagem nas UTI's era a inadequação quanto ao quantitativo de enfermeiros estabelecido pelo Consel ho Federal de Enfermagem. Verificou-se que, por haver um enfermeiro por turno nestas unidades, o mesmo encontrava dificuldades na realização do seu trabal ho e na aplicação do processo de enfermagem como um todo. Esta condição já estava sendo trabalhada pelo enfermei ro gestor das unidades através da solicitação de maior número de enfermeiros junto a chefia de enfermagem.

Tentou-se realizar uma discussão com os enfermeiros sobre estas questões e também sobre o modelo conceitual adotado nas unidades, já que o mesmo expressa as crenças, valores e atitudes profissionais e consequentemente interfere na aplicação do processo de enfermagem mas, por questões internas das unidades isto não foi possível no período em que foi realizado este estudo. Considera-se que esta foi uma barreira, que ainda precisa ser transposta ${ }^{(1)}$.

\section{APLICAÇÃO DA PROPOSTA}

A partir das questões levantadas buscou-se, juntamente com o enfermeiro gestor das unidades, planejar uma proposta de intervenção. Foram apresentadas por ele duas alternativas para se trabalhar: a revisão da prescrição de enfermagem; já que esta é uma fase do processo já implantada, juntamente com o histórico e a evolução de enfermagem ou a implantação da fase de diagnóstico de enfermagem.

A pós conversas com o professor orientador deste estudo, decidiu-se optar por implantar a fase de diagnóstico, apesar de terem sido encontradas deficiências em outras fases, como a de prescrição; pois com a implantação do diagnóstico, o processo, como um todo e as outras fases, poderiam ser trabal hadas paral el amente.

Iniciou-se, então, a construção do impresso de diagnóstico de enfermagem, tendo como base o modelo teórico de Wanda de A guiar Horta já adotado nas unidades, os diagnósticos de enfermagem segundo a N A N DA (2001-2002) e informações obtidas durante o período de observação ${ }^{(1,2)}$.

Esta primeira proposta do impresso ficou muito extensa. Passou-se então, a discussão e elaboração de uma segunda proposta com o professor orientador deste estudo e com o enfermeiro gestor destas unidades. Sugeriu-se ao enfermei ro gestor que o impresso de histórico de enferma- 
gem utilizado nas unidades fosse reformulado e el aborado no mesmo impresso de diagnóstico de enfermagem, visando uma melhor articulação entre estas fases; a sugestão foi por ele aceita.

Esta construção teve como base a abordagem combinada, na qual a conduta cefalocaudal e dos sistemas corporais se integram ${ }^{(5)}$. Foram realizadas várias tentativas na sua construção, até que se chegou a dois model os e a proposta era de que ambos fossem implantados: um para histórico de enfermagem e diagnósticos de admissão e outro para o conjunto diagnóstico, que possibilita um acompanhamento diário da evolução dos mesmos.

Os impressos foram apresentados ao enfermeiro gestor e, devido a paralela reformulação do impresso prescrição de enfermagem pelo referido enfermeiro, decidiu-se que o impresso de conjunto diagnóstico não seria implantado, pois o conjunto de diagnósticos seria incluído no impresso de prescrição de enfermagem e teria espaço para 0 enfermei ro prescrever ao lado de cada diagnóstico levantado, de forma a tornar a prescrição com maior individualização.

Com o término da construção do impresso, o mesmo estava pronto para ser implantado. N este momento, a pedido do enfermeiro gestor, começou a ser elaborado um treinamento sobre o processo de enfermagem a ser realizado com o enfermeiros assistenciais e também a construção de um manual para a sistematização da assistência de enfermagem para as duas UTI's.

0 treinamento com os enfermeiros foi agendado e ocorreu na penúltima semana de estágio nas unidades. Este encontro revelou ser esta uma equipe coesa e interessada por uma mel hor quali dade da assistência, mas que, ao mesmo tempo, ainda encontra dificuldades na implementação do processo de enfermagem como um todo, principal mente na sua documentação, já que a dinâmica da uni dade muitas vezes dificulta esta atividade.

$\mathrm{Na}$ última semana de estágio, busquei acompanhar os enfermeiros assistenciais na aplicação do impresso, visando esclarecer dúvidas que porventura surgissem. N este período percebi que os enfermeiros estavam tendo dificuldades com o manuseio dos diagnósticos de enfermagem, devido a pouca familiaridade dos mesmos com os diagnósticos até o presente momento.

A fase de diagnóstico de enfermagem foi inserida e o impresso está em fase de implantação e será avaliado pelos enfermei ros assistenciais, num período de dois meses, sendo que após este período os próprios enfermeiros farão uma nova aval iação do mesmo e al terações que se façam necessárias.

\section{CONSIDERAÇÕES FINAIS}

Observei que os enfermeiros visualizam a sistematização da assistência de enfermagem como um meio para aplicarem seus conhecimentos técnico-científicos, que ca- racteriza sua prática profissional e conduz a sua autonomia profissional.

Os mesmos ainda encontram dificuldades na sua implementação, visto que, muitas vezes, estes se encontram sobrecarregados com atividades burocráticas, tendo 0 seu exercício profissional dificultado. 0 que surpreendeu foi perceber que há enfermeiros engajados na sua aplicação, dispostos a transpor as dificuldades e que tentam administrar o tempo e realizar suas tarefas com qualidade.

Notei que a instituição e a direção de enfermagem estão abertas e desejosas pela implantação e implementação do processo de enfermagem como um todo não só nestas unidades, mas também em todo o hospital.

A fase inserida proporcionou a utilização do processo de enfermagem como um todo e o impresso elaborado visa facilitar a implementação destas fases nas UTI's, apesar das dificuldades para a sua elaboração e implantação, as quais exigiram empenho, reuniões constantes com o professor orientador e enfermeiro gestor das unidades e revisão da literatura pertinente. A s idas e vindas na sua construção, as dificuldades encontradas e os enfrentamentos inevitáveis produziram um amadurecimento e crescimento profissional, pois, na medida em que foram sendo superados, mostraram-se não serem intransponíveis, mas sim, degraus para o crescimento.

Os objetivos propostos neste estudo foram cumpridos, porém, ainda é preciso maior discussão e estudo do assunto pel os enfermeiros, visto que os mesmos encontram dificuldades na implementação do processo de enfermagem como um todo.

Percebi que, durante o retorno às unidades neste momento de início da implantação, os enfermeiros estão preocupados em aplicar o impresso e tentar entendê-lo; esperase que, após estarem familiarizados com o mesmo, possam não só "preencher o impresso" adequadamente, mas saber articular esta fase de diagnóstico no processo de enfermagem como um todo, para que realmente se torne efetivo.

Cabe ressal tar também que, com a implantação dos diagnósticos de enfermagem nestas uni dades, os enfermeiros devem estar atentos para o perigo do reducionismo, que a proposta dos diagnósticos podem provocar, visto que os padrões estabelecidos pela NANDA não são bem congruentes e a característica dos pacientes internados nestas unidades nem sempre é da forma como se apresenta pela NA NDA ${ }^{(1)}$. Dessa forma, propõe-se que se continuem os estudos e discussões dos diagnósticos de enfermagem, visando uma mel hor adequação dos mesmos nestas unidades.

Também se propõe que se discutam, juntamente com os enfermeiros destas unidades, as questões filosóficas, conceituais e metodológicas que embasam a aplicação do processo, pois se acredita que estas questões, por fundamentarem trabalho do enfermeiro, dirigem a escolha das prioridades que o mesmo real iza no seu processo de trabaIho diante da grande demanda de atividades que unidades 
como estas impõem.

A credita-se que este seja mais um passo da caminhada rumo à melhor qualidade da assistência prestada ao ser humano e conseqüente crescimento profissional para a enfermagem, com val orização e autonomia.

Espera-se que, nesta nova fase da caminhada do processo de enfermagem nestas unidades, os enfermeiros tenham, além de conhecimento e habilidades, “...vontade e ousadia. Vontade para mudar, ousadia para mudar sem temor" (10:26).

ABSTRACT: This paper portraits an experience report related to the application of the nursing process by the nurses and the nursing team, with a proposal of implementing the diagnostic phase. It is a qualitative study, that portraits a systematic, participating, individual and in field observation. It occurred during the discipline Supervised Training II, in two Intensive Care U nits of a University Hospital in the city of Curitiba/ Brazil, in the period of February to J une, 2004. The research showed that the process is applied, but in an incomplete and inarticulate way. It was also verified that the prescription is not seen by the nursing team as an orienting and care-delivery requisite. It was observed the presence of obstacles that hinder the process implementation and need to be overcome, such as the inadequacy concerning the number of nurses established by the Consel ho F ederal de Enfermagem (Nursing Federal Board). A fter the data analysis, it was decided for the implementation of the diagnostic phase and reformulation of the history/file print. It was also carried out a training course with the nurses and a manual of the care-delivery systematic was elaborated. Difficulties were perceived on the part of nurses in applying the diagnostic print and, it is expected that in order to try to overcome them, those nurses keep up with the discussion, aiming not only to fill out the form, but also to be able to articulate it in the process. It is believed to be one more step towards the path that will lead to a better quality care delivery to human beings and consequently valuing and autonomous professional grow th for nurses.

KEY WORDS: Nurse Process; Implantation; Nurse; Diagnosis.

RESUMEN: Este trabajo retrata el relato de experiencia acerca de la aplicación del proceso de enfermería por parte de los enfermeros y del equipo de enfermería con la propuesta de implantación de la fase de diagnóstico. Sucedió durante la asignatura de Pasantía Supervisionada II, en dos universidades de terapia intensiva en un hospital universitario en la ciudad de Curitiba, en el periodo de febrero a junio de 2004. Se trata de un estudio calitativo, que representa una observación sistemática, participante, individual y a campo. La investigación mostró que el proceso es aplicado, sin embargo de manera incompleta y desarticulada. También se ha verificado que la prescripción no es vista por el equipo de enfermería como orientada y que no hay dependencia de ésa para la realización de la asistencia. Se percibió la presencia de obstáculos que impiden la implementación del proceso y que necesitan ser transpuestos, como la inadecuación acerca del cuantitativo de los enfermeros establecidos por el Consejo Federal de Enfermería. Después del análisis de los datos, se decidió por la implantación de la fase de diagnóstico y la reformulación del proceso de impreso histórico. También se realizó un entrenamiento con los enfermeros y un guía de sistematización de asistencia ha sido construido. Se percibió dificultades de los enfermeros en la aplicación del impreso de diagnóstico y se espera que, para intentar superarlas, éstos continúen con las discusiones, con el objetivo de no solamente rellenar papeles, sino saber articularlos en el proceso. Se cree que ese sea más un paso en la caminada en dirección a la mejor cal idad de asistencia prestada al ser humano y en consecuencia crecimiento profesional para enfermería, con valoración y autonomía.

PALABRAS CLAVE: Proceso de Enfermería; Implantación; Diagnostico de Enfermería.

\section{REFERÊNCIAS}

1. Ciaciarullo TI et al. Sistema de assistência de enfermagem: evolução e tendências. São Paulo: Ícone; 2001.

2. Horta WA. Processo de enfermagem. Col. Brigitta E. P. Castellanos. São Paulo: EPU; 1979

3. B ork AM T. Enfermagem de excelência: da visão à ação. Rio de J aneiro: Guanabara K oogan; 2003.

4. Bernocchi-Losey D, Taptich BJ, Iyer PW. Processo de enfermería y diagnóstico de enfermería. 2. ed. Madrid: McGraw-Hill Interamericana; 1993.

5. Talbot L, M eyers-M arquardt M . A valiação em cuidados críticos. 3. ed. (revisão I vone Evangelista Cabral) Rio de J aneiro: Reichman eA ffonso; 2001.

6. Carpenito LJ. Planos de cuidados de enfermagem e documentação: diagnóstico de enfermagem e problemas colaborativos. 2. ed. Porto A legre: A rtes M édicas; 1999.

7. Farias JN, Nobrega M M L, Perez V LA B, Coler MS. Diagnóstico de enfermagem - uma abordagem conceitual e prática. J oão Pessoa, Santa M arta, 1990.

8. Timby BK. Conceitos e habilidades fundamentais no atendimento de enfermagem. 6 ed. Porto A legre: A rtmed, 2001.

9. Carraro TE, Westphalen MEA, Zagonel IS. M etodologias para assistência de enfermagem - teorizações, modelos e subsídios para a prática. Goiânia: A B, 2001. Org. M ary E. A. Westphalen.

ENDEREÇO DOSAUTORES: Rua Trajano Reis, 248 - ap. 84 Curitiba - PR 80510-220

enferdani2004@yahoo.com.br 